This item was submitted to Loughborough's Institutional Repository (https://dspace.lboro.ac.uk/) by the author and is made available under the following Creative Commons Licence conditions.

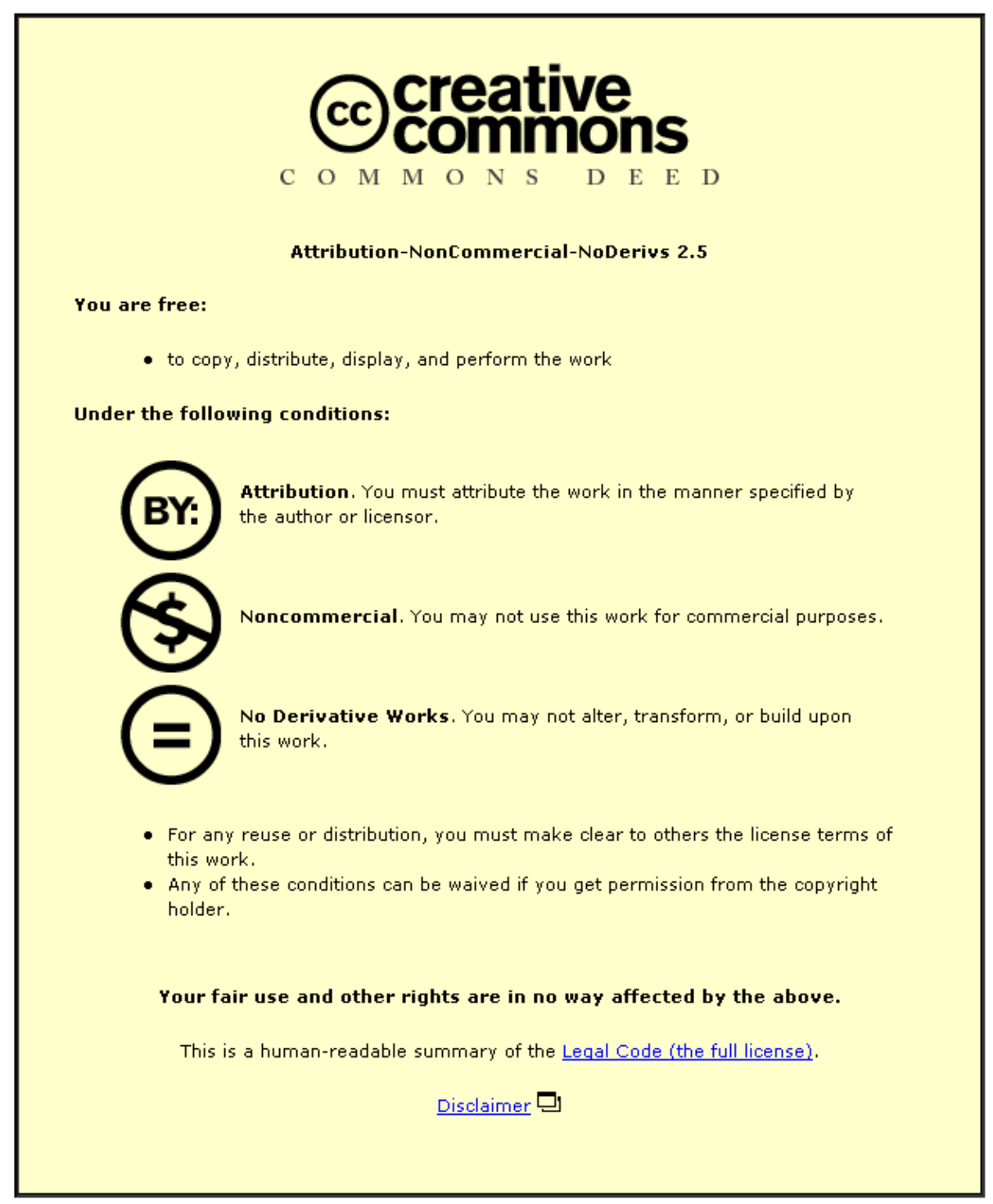

For the full text of this licence, please go to: http://creativecommons.org/licenses/by-nc-nd/2.5/ 


\title{
The margin for error when releasing the high bar for dismounts
}

\author{
M.J. Hiley and M.R. Yeadon \\ School of Sport and Exercise Sciences, Loughborough University, Loughborough, UK
}

\begin{abstract}
In Men's Artistic Gymnastics the current trend in elite high bar dismounts is to perform two somersaults in an extended body shape with a number of twists. Two techniques have been identified in the backward giant circles leading up to release for these dismounts (Arampatzis and Brüggemann, 1999). At the Sydney 2000 Olympic Games 95\% of gymnasts used the "scooped" backward giant circle technique rather than the "traditional" technique. It was speculated that the advantage gained from the scooped technique was an increased margin for error when releasing the high bar. A four segment planar simulation model of the gymnast and high bar was used to determine the margin for error when releasing the bar from performances at the Sydney 2000 Olympic Games. The eight high bar finalists and three gymnasts using the traditional backward giant circle technique were chosen for analysis. Model parameters were optimised to obtain a close match between the simulation and the actual performance in terms of rotation angle $\left(1.2^{\circ}\right)$, bar displacements $(0.014 \mathrm{~m})$ and release velocities (2\%). The matched simulation was used to determine the time window around the actual point of release for which the model had appropriate release parameters to complete the dismount successfully. The scooped backward giant circle technique, used by the majority of elite gymnasts, resulted in a greater margin for error (release window 88 - $157 \mathrm{~ms}$ ) when releasing the bar compared with the traditional technique (release window 73 - $84 \mathrm{~ms}$ ).
\end{abstract}

Keywords : gymnastics, high bar, simulation, dismounts, release window

\section{Introduction}

In Men's Artistic Gymnastics two distinct techniques have been identified in the accelerated backward giant circles used prior to a double layout somersault dismount (Figure 1) from the high bar (Arampatzis and Brüggemann, 1999). These may be referred to as 'traditional' and 'scooped' backward giant circles (Figure 2). In the traditional technique the gymnast extends his body close to the highest point of the circle whereas in the scooped technique he maintains the angles at the hip and shoulder joints through the highest point and extends during the downswing (Figure 2).

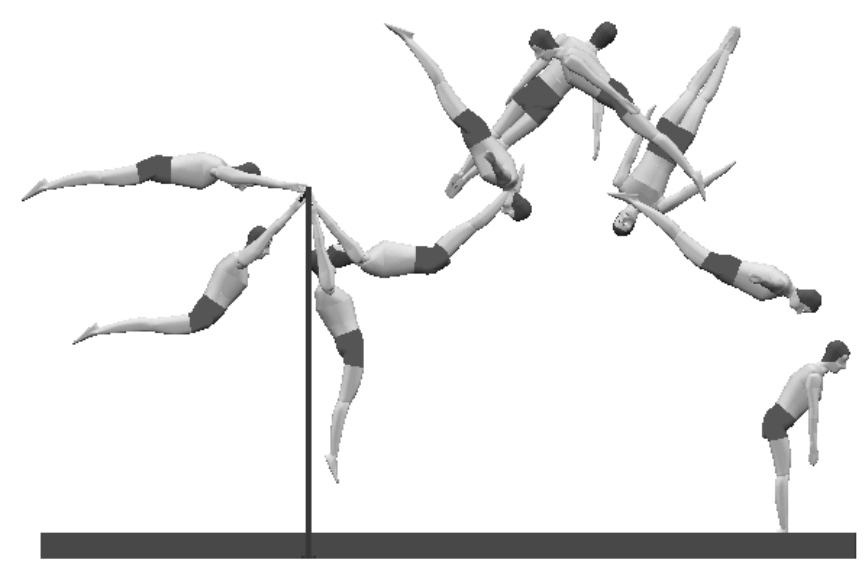

Figure 1. A double layout somersault dismount from high bar with a full twist. 


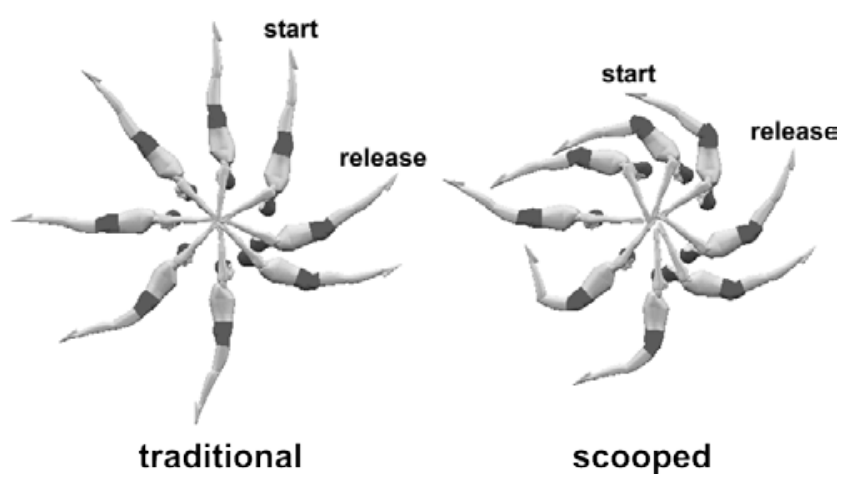

Figure 2. The two techniques (traditional and scooped) used by elite gymnasts during accelerated backward giant circles prior to a double layout somersault dismount.

The requirements of a successful dismount are that the gymnast has sufficient angular momentum and flight time to complete the required number of somersaults and travels safely away from the apparatus (Brüggemann et al., 1994). The flight and angular momentum of the gymnast are determined at release and are a result of the gymnast's technique during the preceding backward giant circle. At the Sydney 2000 Olympic Games only three of the 68 gymnasts who performed double layout dismounts in the qualifying competition used a traditional accelerated backward giant circle prior to releasing the bar. This strongly suggests that there must be some advantage to using a scooped technique. Arampatzis and Brüggemann (1999) calculated the normalised angular momentum during flight from 29 double layout somersault dismounts, nine using the traditional technique and 20 using the scooped technique, and found no significant difference between the two techniques. It is likely therefore that gymnasts select the scooped technique for reasons other than the production of angular momentum.

It is speculated that the scooped accelerated giant circle leads to an increased margin for error when releasing the bar compared with the traditional technique. The margin for error may be quantified in terms of the release window during which the gymnast has suitable linear and angular momentum for performing the dismount. If the gymnast releases at any point during this window he will have sufficient flight and rotation to complete the dismount.

The aim of this study is to determine the release window for gymnasts performing double layout somersault dismounts from traditional and scooped accelerated backward giant circles at the Sydney 2000 Olympic Games. In addition the relationship between the configuration of the gymnast when passing through the highest and lowest points of the giant circle and the size of the release window will be investigated.

\section{Methods}

All high bar performances from the Sydney 2000 Olympic games were recorded using two digital video cameras (Sony Digital Handycam DCR-VX1000E), operating at $50 \mathrm{~Hz}$ with shutter speeds of $1 / 600 \mathrm{~s}$. The two cameras were located $8 \mathrm{~m}$ above the landing surface and $27 \mathrm{~m}$ and $37 \mathrm{~m}$ from the high bar. Prior to the start of each competition a calibration structure comprising 48 spheres of diameter $0.10 \mathrm{~m}$ spanning a volume measuring $3 \mathrm{~m} \times 5 \mathrm{~m} \times 5 \mathrm{~m}$ was positioned with its centre at the midpoint of the high bar and was recorded by both cameras. The high bar was loaded statically in order to estimate the vertical stiffness coefficient. A $0.10 \mathrm{~m}$ diameter sphere was positioned at the midpoint of the bar which was loaded to $2245 \mathrm{~N}$. The loading of the bar was 
video recorded together with a calibration structure surrounding the centre of the bar. The stiffness coefficient was calculated using Hooke's law.

The centres of the calibration spheres were digitised in five video fields from both camera views. The performances of the eight highest scoring gymnasts who reached the high bar apparatus final and the three gymnasts who used a traditional backward giant circle technique were selected for analysis. The last $3 / 4$ backward giant circle and the dismount were digitised for each subject. In each of the movement fields the centre of the hand, elbow, shoulder, hip, knee and ankle joint centres and toes on each side of the body were digitised along with the centre of the gymnast's head and the centre of the high bar between the gymnast's hands. The data obtained from digitising the images of the calibration spheres and their known locations were used to calculate the 11 Direct Linear Transformation parameters for each of the cameras (Abdel-Aziz and Karara, 1971). The two sets of digitised movement data were synchronised using the method of Yeadon and King (1999). Synchronised digitised coordinate data from each camera view along with the camera parameters were used to reconstruct the three-dimensional locations of the body landmarks using the Direct Linear Transformation. Joint angles for the left and right sides were averaged to produce input for a planar simulation model. Quintic splines (Wood and Jennings, 1979) were used to fit the orientation and joint angle time histories so that derivatives could be obtained (Yeadon, 1990a).

A set of anthropometric measurements of a "mean" elite gymnast was obtained as the mean measurements taken from seven National Squad gymnasts. These measurements were scaled for each of the 11 competitors using segments lengths and widths obtained from the video digitisation and inertia parameters were calculated using the model of Yeadon (1990b). The angular momentum about the mass centre during the dismount was calculated for each competitor. The time of flight was determined from the field before there was clear space between the gymnast's hands and the bar and the field before contact between the gymnast's feet and the landing mat. The horizontal and vertical displacements of the mass centre during flight were used to calculate the horizontal and vertical velocity at release using a least squares fit and assuming constant acceleration.

A four segment planar model of a gymnast comprising arm, torso, thigh and lower leg segments was used to simulate the movement around the bar (Yeadon and Hiley, 2000). The high bar and the gymnast's shoulder structure were modelled as damped linear springs (Figure 3).

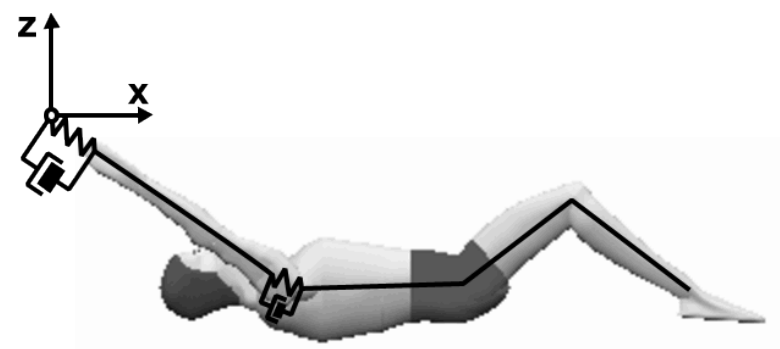

Figure 3. The four segment gymnast - high bar simulation model with damped springs representing bar and shoulder elasticity.

Input to the simulation model comprised the segmental inertia parameters, the stiffness and damping coefficients of the bar and shoulder springs, the initial displacement and velocity of the bar, the initial angular velocity of the arm, the initial orientation of the arm and the joint angle time histories in the form of quintic splines 
obtained from the video analysis. Output from the model comprised the time histories of the horizontal and vertical bar displacements, the linear and angular momentum of the model and the rotation angle $\phi$ (the angle from the vertical of the line joining the neutral bar position to the mass centre).

The equations of motion were derived using Newton's Second Law and by taking moments about the neutral bar position and the segment mass centres. The angular momentum of the body about its mass centre was calculated using:

$$
\mathrm{h}=\sum_{\mathrm{i}=1}^{4}\left(\mathrm{I}_{\mathrm{i}} \dot{\phi}_{\mathrm{i}}+\mathrm{m}_{\mathrm{i}}\left(\dot{\mathrm{Z}}_{\mathrm{i}} \mathrm{X}_{\mathrm{i}}-\dot{\mathrm{X}}_{\mathrm{i}} \mathrm{Z}_{\mathrm{i}}\right)\right)
$$

where $\mathrm{X}_{\mathrm{i}}=\left(\mathrm{x}_{\mathrm{i}}-\mathrm{x}_{\mathrm{cm}}\right), \mathrm{Z}_{\mathrm{i}}=\left(\mathrm{z}_{\mathrm{i}}-\mathrm{z}_{\mathrm{cm}}\right),\left(\mathrm{x}_{\mathrm{cm}}, \mathrm{z}_{\mathrm{cm}}\right)$ = whole body mass centre location, $\mathrm{m}_{\mathrm{i}}=$ segmental mass, $\mathrm{I}_{\mathrm{i}}=$ segmental moment of inertia, $\dot{\phi}_{\mathrm{i}}=$ segmental angular velocity.

The angular momentum was normalised by dividing by $2 \pi$ times the moment of inertia of the body about its mass centre when straight and multiplying by the flight time to give the equivalent number of straight somersaults in the subsequent flight phase. The time of flight of a simulation was calculated from the release and landing heights of the mass centre and the vertical velocity at release using the equation for constant acceleration under gravity. The height of the mass centre on landing was taken from the video analysis of each gymnast.

In order to determine the release window using the simulation model a close match between the simulated and actual performance was required. The simulation model was implemented with the Simulated Annealing optimisation algorithm (Goffe et al., 1994). A cost function $\mathrm{F}$ was established to minimise the difference between the recorded performance from the video analysis and a simulation of this performance. The weightings of the cost function $\mathrm{F}$ are shown in equation (2).

$$
\mathrm{F}=10 \phi+400\left(\mathrm{x}_{\mathrm{b}}+\mathrm{z}_{\mathrm{b}}\right)+20\left(\mathrm{~h}+\dot{\mathrm{x}}_{\mathrm{cm}}+\dot{\mathrm{z}}_{\mathrm{cm}}\right)+\phi_{\mathrm{o}}
$$

where $\phi=$ root mean squared (rms) difference in degrees between recorded and simulated rotation angle, $\mathrm{x}_{\mathrm{b}}, \mathrm{z}_{\mathrm{b}}=$ the rms differences between recorded and simulated bar displacements, $\mathrm{h}=$ absolute difference in normalised angular momentum at release between simulation and actual performance, $\dot{\mathrm{x}}_{\mathrm{cm}}, \dot{\mathrm{z}}_{\mathrm{cm}}=$ absolute differences in linear velocity at release between simulation and actual performance, $\phi_{0}=$ absolute difference in initial rotation angle between simulation and actual performance. The weightings were chosen so that each of the six components of the cost function made approximately equal contributions.

Since the aim of the matching process was to provide good agreement between the simulation and the actual performance leading up to release only the last $180^{\circ}$ of the final giant circle was simulated. The subject specific inertia parameters calculated for each of the gymnasts in the video analysis were used in the simulation model. The initial conditions, including the initial angle, angular velocity and bar displacements, for each simulation were taken from the video analysis. The initial estimate of the bar stiffness coefficient for the bar was determined from the static loading of the bar. During the optimisation the following parameters were allowed to vary in order to improve the match between the recorded and simulated performance. Bar stiffness was allowed to vary between $20000 \mathrm{~N} \cdot \mathrm{m}^{-1}$ and $27500 \mathrm{~N} . \mathrm{m}^{-1}$ to conform with the specifications of the International Gymnastics Federation (FIG, 2000). The damping 
coefficient of the bar was allowed to vary between 0 N.s.m ${ }^{-1}$ and 500 N.s.m ${ }^{-1}$. The stiffness and damping coefficients of the shoulder spring were allowed to vary over wider ranges than those of the bar springs. The masses of the arms and legs were independently allowed to vary by $\pm 25 \%$ and the torso mass was adjusted to maintain the whole body mass of the gymnast. In addition small variations in the initial conditions were permitted to compensate for any errors produced in their calculation.

Once the optimisation procedure had provided a simulation to match the video performance of the final $180^{\circ}$ of rotation leading up to the release, the matching simulation for each gymnast was continued beyond the point of release so that a release window could be determined. It was assumed that the gymnast maintained contact with the high bar and continued with the same joint angle changes as occurred after release. The release window was defined as the period of time for which the model possessed angular momentum within $\pm 10 \%$ of the actual release value, landed with the mass centre between $1.0 \mathrm{~m}$ and $3.5 \mathrm{~m}$ from the bar and had a time of flight of at least $1.2 \mathrm{~s}$. In addition the normalised angular momentum was not allowed to drop below 1.41 straight somersaults which was the lowest value reported by Kerwin et al. (1990) for double layout somersault dismounts. The angular momentum limits of $\pm 10 \%$ were chosen so that the gymnast would be able to make compensatory configurational changes in flight and successfully land the double layout somersault dismount. This was investigated using a simulation model of aerial movement (Yeadon et al., 1990) for one of the performances. The release window was allowed to start before and end after the actual release of the gymnast so long as the above requirements were satisfied.

The change in the rotation angle and the change in the direction of the mass centre velocity over the release window were compared for traditional and scooped circles. The relationship between gymnast configuration and magnitude of release window was determined by regressing the sum of the angles at the hip and shoulder joints at the highest point of the final giant circle against the size of the release window. This was repeated for the lowest point of the giant circle. The highest and lowest points of the giant circle occurred when the gymnast's mass centre passed directly above or below the neutral bar position, respectively.

\section{Results}

The reconstruction error for the $3 \mathrm{~m} \times 5 \mathrm{~m} \times 5 \mathrm{~m}$ calibration volume was calculated to be $0.013 \mathrm{~m}$, with the field of view spanning over $8 \mathrm{~m}$. The reconstruction error for the calibration volume used in the calculation of the bar stiffness coefficient was $0.002 \mathrm{~m}$. The initial estimate of the bar stiffness coefficient was calculated from the static loading as 26855 N.m ${ }^{-1}$ which lies within the specified FIG norms (FIG, 2000).

The values for the normalised angular momentum and the vertical velocity at release were similar for the traditional and the scooped backward giant circles (Table 1) and are comparable with the values reported by Aramptzis and Brüggemann (1999). However, the horizontal mass centre velocities of the traditional giant circles at release were all larger than those of the scooped circles. 
Table 1. Velocity of the mass centre and angular momentum about the mass centre at release for scooped and traditional techniques

\begin{tabular}{|c|c|c|c|}
\hline $\begin{array}{l}\text { competitor } \\
\text { (no.) }\end{array}$ & $\begin{array}{c}\text { horizontal } \\
\text { velocity } \\
\left(\mathrm{m} \cdot \mathrm{s}^{-1}\right)\end{array}$ & $\begin{array}{c}\text { vertical } \\
\text { velocity } \\
\left(\mathrm{m}^{-1} \mathrm{~s}^{-1}\right)\end{array}$ & $\begin{array}{l}\text { angular momentum } \\
\text { (straight somersaults) }\end{array}$ \\
\hline 126 & 1.16 & 4.70 & 1.63 \\
\hline 132 & 0.92 & 4.62 & 1.57 \\
\hline 147 & 1.16 & 4.68 & 1.65 \\
\hline 157 & 1.66 & 4.97 & 1.61 \\
\hline 174 & 1.69 & 4.78 & 1.73 \\
\hline 176 & 1.19 & 5.38 & 1.72 \\
\hline 185 & 1.22 & 4.57 & 1.59 \\
\hline 190 & 1.14 & 4.97 & 1.68 \\
\hline mean & 1.27 & 4.83 & 1.65 \\
\hline $155^{*}$ & 1.96 & 4.83 & 1.45 \\
\hline $180^{*}$ & 2.30 & 4.94 & 1.66 \\
\hline $182 *$ & 2.08 & 4.89 & 1.64 \\
\hline mean & 2.11 & 4.89 & 1.58 \\
\hline
\end{tabular}

* indicates a traditional backward giant circle technique

Over the 11 performances studied, the simulation model was able to match the recorded rotation angle during the final $180^{\circ}$ leading up to release to within $1.2^{\circ} \mathrm{rms}$ difference, and the horizontal and vertical displacements of the bar to within $0.014 \mathrm{~m}$ rms difference (Figure 4). The simulation model matched the normalised angular momentum and the linear velocities at release to within 2\%. For the 11 performances the average stiffness coefficient of the bar obtained in the matching procedure was 26129 N.m ${ }^{-1}$, less than $3 \%$ different from the static loading value. The average damping coefficient for the bar was 174 N.s.m ${ }^{-1}$. 
(a)

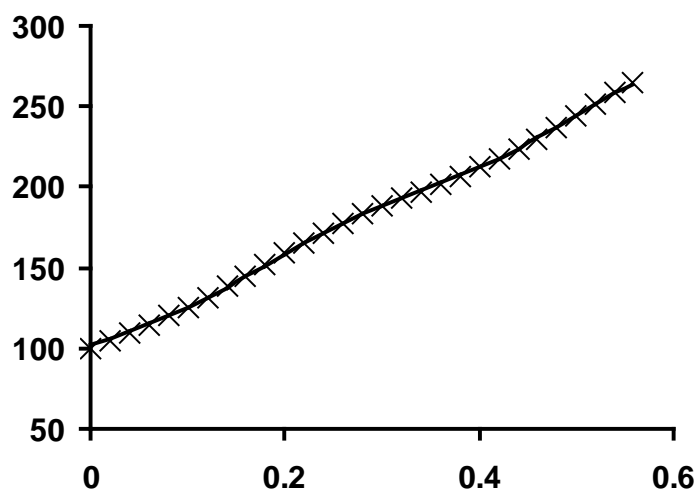

(b)

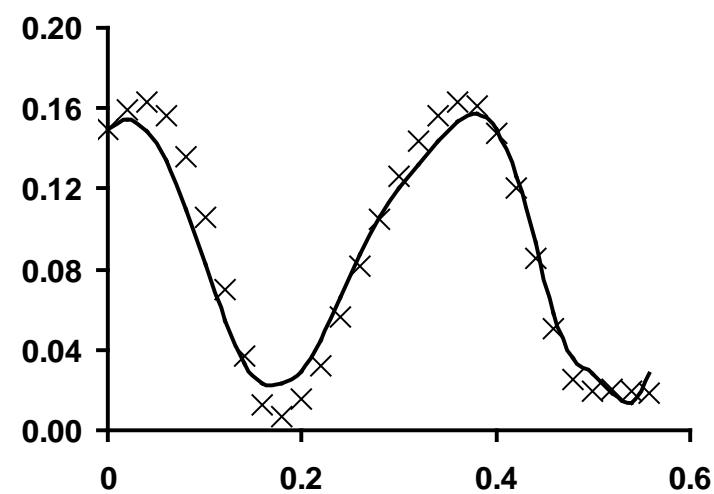

Figure 4. Typical matches between simulation (solid line) and actual performance (crosses) of (a) whole body rotation angle and (b) bar displacements.

Using the simulation model of aerial movement it was found that it was possible to successfully complete the double layout somersault dismount by making small configurational changes when the angular momentum in flight was either increased or decreased by $10 \%$. To cope with the extra rotation competitor 176 would have to change his hip angle during flight by less than $10^{\circ}$ and elevate his arms by an additional $40^{\circ}$ during the mid-section of the flight phase. Similarly to cope with the reduced rotation the same gymnast would have to lower his arms to his sides during the midsection of the flight phase.

The release windows determined by simulation for the eight scooped circles were all greater than the release windows of the three traditional circles (Table 2). The average release window for the gymnasts using a scooped technique was 117 milliseconds (range 88 - $157 \mathrm{~ms}$ ) whereas the average release window for the gymnasts using a traditional technique was $79 \mathrm{~ms}$ (range 73 - $84 \mathrm{~ms}$ ). 
Table 2. Sum of the hip and shoulder angles at the highest and lowest points of the giant circle

\begin{tabular}{|c|c|c|c|c|}
\hline $\begin{array}{c}\text { competitor } \\
\text { (no.) }\end{array}$ & $\begin{array}{c}\text { sum of } \\
\text { angles at } \\
\text { highest } \\
\text { point } \\
\left({ }^{\circ}\right)\end{array}$ & $\begin{array}{c}\text { sum of } \\
\text { angles at } \\
\text { lowest } \\
\text { point } \\
\left({ }^{\circ}\right)\end{array}$ & $\begin{array}{c}\text { release } \\
\text { window } \\
(\mathrm{ms})\end{array}$ & $\begin{array}{c}\text { release } \\
\text { window } \\
\left({ }^{\circ}\right)\end{array}$ \\
\hline 126 & 122 & -52 & 157 & 55 \\
\hline 132 & 135 & -36 & 104 & 36 \\
\hline 147 & 143 & -39 & 103 & 37 \\
\hline 157 & 146 & -40 & 96 & 36 \\
\hline 174 & 119 & -40 & 101 & 39 \\
\hline 176 & 124 & -47 & 150 & 53 \\
\hline 185 & 132 & -39 & 135 & 52 \\
\hline 190 & 78 & -25 & 88 & 29 \\
\hline mean & $\mathbf{1 2 5}$ & $\mathbf{- 4 0}$ & $\mathbf{1 1 7}$ & $\mathbf{4 2}$ \\
\hline & & & & \\
\hline $155^{*}$ & 9 & -12 & 80 & 27 \\
\hline $180^{*}$ & -15 & -6 & 73 & 24 \\
\hline $182^{*}$ & 32 & -19 & 84 & 25 \\
\hline mean & $\mathbf{9}$ & $\mathbf{- 1 2}$ & $\mathbf{7 9}$ & $\mathbf{2 5}$ \\
\hline
\end{tabular}

* indicates a traditional backward giant circle technique

Note: (a) hip and shoulder angles are defined away from a handstand position (see Figure 5)

(b) last column expresses release window in terms of the change in rotation angle

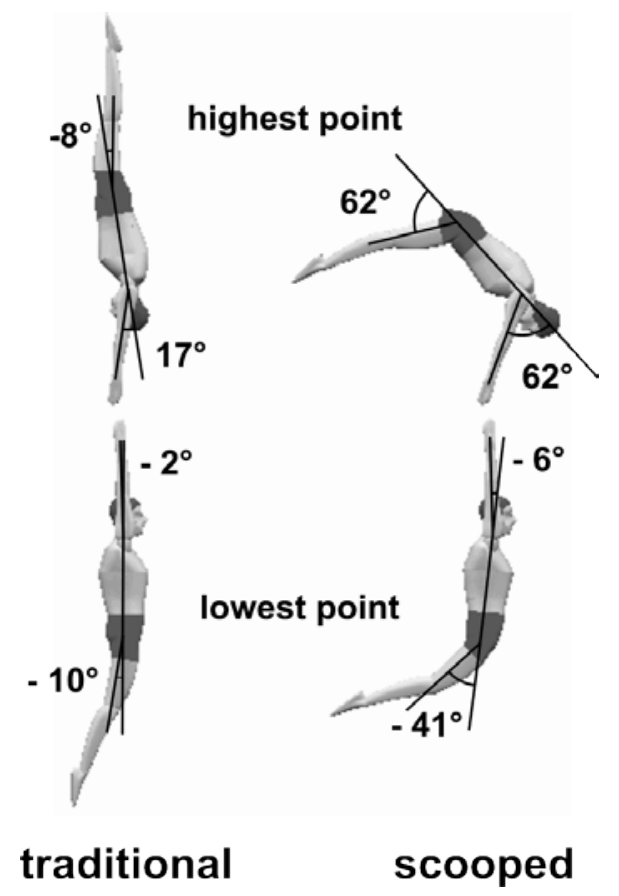

Figure 5. Typical configurations of the gymnasts passing through the highest and lowest points of the final giant circle leading up to release using a traditional technique and a scooped technique. The more hyper-extended configuration of the scooped technique at the lowest point is associated with a larger release window. 
There was only a weak relationship between the sum of the joint angles at the highest point of the final $3 / 4$ giant circle and the size of the release window $\left(R^{2}=0.40, p=0.04\right)$. At the lowest point the relationship was stronger $\left(\mathrm{R}^{2}=0.71, \mathrm{p}=0.001\right)$. Typical configurations as the gymnast passes through the highest and lowest point for a traditional and a scooped backward giant circle are shown in Figure 5. The path of the mass centre during the final $3 / 4$ giant circle for a traditional and a scooped backward giant circle are quite different (Figure 6).

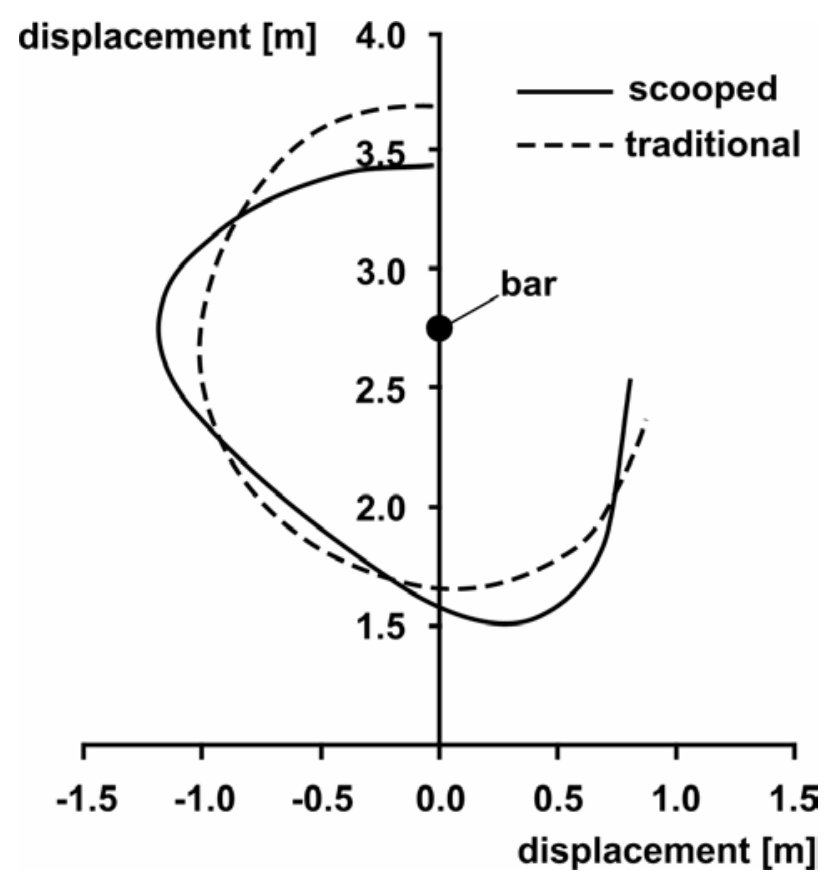

Figure 6. The path of the mass centre during the last backward giant circle leading up to release for one gymnast using a traditional technique and one gymnast using a scooped technique. The flatter final part of the scooped path results in a more consistent flight trajectory and a larger release window.

\section{Discussion}

Computer simulation provided a means for addressing hypothetical scenarios of different release times. The close agreement between simulation and actual performance gives a measure of confidence in the results. This approach is limited, however, by the assumption of configuration changes when releasing later than in the actual performance and also by the somewhat arbitrary criteria for a successful dismount. While the calculated release windows would change slightly if different assumptions were made the relationship between technique and release window would be unchanged.

The reason why the scooped accelerated backward giant circle produced a larger release window may be understood by looking at the path of the mass centre during the final $3 / 4$ giant circle. The mass centre path for a traditional technique (dashed line) and a scooped technique (solid line) are shown in Figure 6. In the traditional technique the path of the mass centre is almost circular and the velocity direction changes rapidly as the gymnast approaches release. This explains why the gymnasts using the traditional technique tended to have greater horizontal velocity at release compared to the gymnasts using the scooped technique (Table 1). In the scooped technique there is a flattening of the path of the mass centre and the velocity direction changes less rapidly 
as the gymnast approaches release. The mean change in the direction of mass centre velocity during the release window was $22.8^{\circ} \pm 1.4^{\circ}$ for the traditional technique and $10.3^{\circ} \pm 6.2^{\circ}$ for the scooped technique. The corresponding changes in rotation angle over the release window had mean values of $25.3^{\circ} \pm 1.5^{\circ}$ (traditional) and $42.1^{\circ} \pm 9.7^{\circ}$ (scooped). The circular mass centre path of the traditional circles led to the release window being limited primarily by the direction of the mass centre velocity. In contrast the flattened mass centre path of the scooped circles posed less constraints with regard to velocity direction, the lower limit of the release window being determined primarily by the angular momentum upper limit.

When the bar displacements are subtracted from the mass centre locations the flattening of the path of the mass centre is less pronounced. The movement of the bar, therefore, makes a noticeable contribution to the flattening of the mass centre path. However, since the loading of the bar is dependent on the configurational changes of the gymnast no quantification of the contributions due to the bar displacements and the configurational changes has been attempted.

Although there were only three traditional circles and so no tests for significant differences between the windows of the two techniques were carried out, it is striking that the release windows of the eight scooped circles were all greater than those of the three traditional circles. Even so the regression analysis showed that the release window was only weakly related to the configuration at the highest point. This may simply be interpreted that using a scooped giant circle leads to a larger release window and that the amount the angles at the hip and shoulder are closed at the highest point is not critical. The relationship between the configuration at the lowest point and the release window was much stronger. The larger release windows were associated with a more hyper-extended configuration through the lowest point (Figure 5). It is likely that the configuration the gymnast adopts through the highest point is used to help the gymnast achieve the hyper-extended configuration through the lowest point.

The larger release windows associated with the scooped technique allow a gymnast a greater margin for error in timing the release for a dismount and may be expected to lead to a more consistent performance. Since Artistic Gymnastics is a sport in which timing and consistency are important factors for success it may be expected that there will be other instances of elite technique that optimise the margin for error of a skill.

\section{Acknowledgement}

The authors wish to acknowledge the support of the British Gymnastics World Class Programme, Sport England, UK Sport, Pfitzer and the International Olympic Committee Medical Commission.

\section{References}

Abdel-Aziz, Y.I., and Karara, H.M. 1971. Direct linear transformation from comparator coordinates into object space coordinates in close-range photogrammetry (ASP Symposium on Close-Range Photogrammetry). Falls Church, VA: American Society of Photogrammetry. pp. 1-18.

Arampatzis, D. and Brüggemann, G-P. 1999. Mechanical and energetic processes during the giant swing exercise before dismount and flight elements on the high bar and uneven parallel bars. Journal of Biomechanics 32, 811-820.

Brüggemann, G-P., Cheetham, P.J., Alp, Y. and Arampatzis, D. 1994. Approach to a biomechanical profile of dismounts and release-regrasp skills of the high bar. Journal of Applied Biomechanics 10, 291-312. 
Fédération Internationale de Gymnastique 2000. Manual apparatus norms. Part IV testing procedures. F.I.G. Switzerland.

Goffe, W.L., Ferrier, G.D. and Rogers, J. 1994. Global optimisation of statistical functions with simulated annealing. Journal of Econometrics 60, 65-99.

Kerwin, D.G., Yeadon, M.R. and Lee, S.C. 1990. Body configuration in multiple somersault high bar dismounts. International Journal of Sport Biomechanics 6, 147156.

Wood, G.A. and Jennings, L.S. 1979. On the use of spline functions for data smoothing. Journal of Biomechanics 12, 477-479.

Yeadon, M.R. 1990a. The simulation of aerial movement-I. The determination of orientation angles from film data. Journal of Biomechanics 23, 59-66.

Yeadon, M.R. 1990b. The simulation of aerial movement-II. A mathematical inertia model of the human body. Journal of Biomechanics 23, 67-74.

Yeadon, M.R., Atha, J. and Hales, F.D. 1990c. The simulation of aerial movement-IV. A computer simulation model. Journal of Biomechanics 23, 85-89.

Yeadon, M.R., and Hiley, M.J. 2000. Mechanics of the backward giant circle on the high bar. Human Movement Science 19, 153-173.

Yeadon, M.R. and King, M.A. 1999. A method for synchronising digitised video data. Journal of Biomechanics 32, 983-986. 